\title{
Article \\ Nonlinear Analysis of Tropical Waves and Cyclogenesis Excited by Pressure Disturbance in Atmosphere
}

\author{
Zi-Liang Li $^{1, *(D)}$ and Jin-Qing Liu ${ }^{2,3,4}$ \\ 1 College of Oceanic and Atmospheric Sciences, Ocean University of China, Qingdao 266100, China \\ 2 Hunan Meteorological Observatory, Changsha 410118, China; liujq_0912@126.com \\ 3 Institute of Tropical and Marine Meteorology/Guangdong Provincial Key Laboratory of Regional Numerical \\ Weather Prediction, CMA, Guangzhou 510641, China \\ 4 Heavy Rain and Drought Flood Disasters in Plateau and Basin Key Laboratory of Sichuan, \\ Chengdu 610072, China \\ * Correspondence: liziliang@ouc.edu.cn
}

Citation: Li, Z.-L.; Liu, J.-Q.

Nonlinear Analysis of Tropical Waves and Cyclogenesis Excited by Pressure Disturbance in Atmosphere.

Mathematics 2021, 9, 3038. https:// doi.org/10.3390/math9233038

Academic Editor: Andrey Amosov

Received: 13 October 2021

Accepted: 22 November 2021

Published: 26 November 2021

Publisher's Note: MDPI stays neutral with regard to jurisdictional claims in published maps and institutional affiliations.

Copyright: (๑) 2021 by the authors. Licensee MDPI, Basel, Switzerland. This article is an open access article distributed under the terms and conditions of the Creative Commons Attribution (CC BY) license (https:// creativecommons.org/licenses/by/ $4.0 /)$.

\begin{abstract}
The horizontal equations of motion for an inviscid homogeneous fluid under the influence of pressure disturbance and waves are applied to investigate the nonlinear process of solitary waves and cyclone genesis forced by a moving pressure disturbance in atmosphere. Based on the reductive perturbation analysis, it is shown that the nonlinear evolution equation for the wave amplitude satisfies the Korteweg-de Vries equation with a forcing term (fKdV equation for short), which describes the physics of a shallow layer of fluid subject to external pressure forcing. Then, with the help of Hirota's direct method, the analytic solutions of the fKdV equation are studied and some exact vortex solutions are given as examples, from which one can see that the solitary waves and vortex multi-pole structures can be excited by external pressure forcing in atmosphere, such as pressure perturbation and waves. It is worthy to point out that cyclone and waves can be excited by different type of moving atmospheric pressure forcing source.
\end{abstract}

Keywords: tropical cyclogenesis; pressure disturbance; fKdV equation; bilinear direct method; multi-pole vortex

\section{Introduction}

Constructing the nonlinear model and its exact solutions play a major role in describing complex phenomenon in atmospheric and oceanic fluid mechanics. Several methods are available to obtain exact solutions of nonlinear equations, such as the inverse scattering method, bilinear method, sine-cosine method, Jacobi elliptic function method, and the homotopy perturbation method [1-3]. The bilinear direct method can be regarded as the effective technique to investigate nonlinear equation integrability, this method can produce quasi periodic wave solutions, rational solutions, lump solutions, multi-soliton solutions, and other exact solutions by converting a nonlinear equation into a bilinear form under a dependent variable transformation [4]. Recently, the Bell polynomial method can simply derive the bilinear form of a nonlinear model due to the relationship between Bell polynomial and bilinear form [5]. Based on the bilinear formulation, a generalized Wronskian technique was proposed to determine the exact solutions of the KdV equation and forced $\mathrm{KdV}$ equations [2].

The KdV equation includes weak nonlinearity and dispersion and characterizes some interesting phenomenon including interaction of solitary wave and stratified internal waves. A mathematical model describing tsunami generation influenced by submarine landslides was demonstrated as

$$
\frac{\partial \Phi}{\partial t}+e_{1} \Phi \frac{\partial \Phi}{\partial x}+e_{2} \frac{\partial^{3} \Phi}{\partial y^{3}}+e_{3} \frac{\partial \Phi}{\partial x}=\frac{\partial S}{\partial x}
$$


where $\Phi$ represents the elevation of water surface and $S$ denotes bottom. The classical KdV equation can be reduced by vanishing the forcing term, which is known to be integrable, whereas the forced KdV equation is unknown to be integrable. Recently, several methods, such as the exact three-wave method and bilinear direct method, were implemented to construct the solutions of $\mathrm{fKdV}$ equation [6-12]. The forced $\mathrm{KdV}$ equation is also used to describe internal wave forced by topography in geophysical fluids as well as the moving debris induced excitations in plasmas [13-15].

The idea that pressure waves can trigger cyclone genesis is not new [16-18]. It has been known that hurricanes can form from African pressure waves and the Madden-Julian oscillation modulates north Pacific tropical cyclone activity [17]. The pressure waves play major roles in causing tropical cyclone genesis by increasing moisture convection and the low-level vorticity as well as altering the local vertical shear pattern. Some types of waves, such as mixed Rossby gravity waves and Kelvin waves, may modulate the wind fields and the tropical rainfall in low-pressure regions, where wave energy tends to accumulate $[19,20]$. The pressure waves can alter the mean flow and sea surface temperatures to have some effect on cyclone genesis [21]; however, despite interesting results for fKdV equation being obtained, they have not been applied in exploring the relationship between the pressure disturbance and cyclone genesis cases. Here, we aim to fill this gap by constructing the forced $\mathrm{KdV}$ equation to investigate the cyclone genesis excited by pressure disturbance. In this work, from the perspective of energy conservation, a novel method to be proposed to derive the $\mathrm{fKdV}$ equation by introducing the pressure disturbance to the mathematical model. The solitary wave and vortex solution of a forced KdV equation are obtained by bilinear direct method, which can explain the relationship between the pressure disturbance and cyclone genesis.

The organization of this article is as follows. In Section 2, a nonlinear mathematical model is constructed to study vortex and waves excited by pressure forcing source in fluid, and the $\mathrm{KdV}$ equation with pressure forcing term is derived. The bilinear direct method is applied on the forced $\mathrm{KdV}$ equation, and the exact vortex and wave solutions are exploited and graphically represented in Section 3. Finally, discussion and concluding remarks are given in Section 4.

\section{Constructing Nonlinear Mathematical Model}

The governing equation is an incompressible homogeneous fluid with a constant density. The vertical acceleration of the flow is assumed to be small so that the equation of motion in the vertical $z$-direction reduces to the hydrostatic equation. The horizontal equations of motion including Earth rotation are given by

$$
\begin{gathered}
\frac{\partial u}{\partial t}+u \frac{\partial u}{\partial x}+v \frac{\partial u}{\partial y}-f v+\frac{1}{\rho_{0}} \frac{\partial p}{\partial x}=0 \\
\frac{\partial v}{\partial t}+u \frac{\partial v}{\partial x}+v \frac{\partial v}{\partial y}+f u+\frac{1}{\rho_{0}} \frac{\partial p}{\partial y}=0 \\
\frac{\partial u}{\partial x}+\frac{\partial v}{\partial y}+\frac{\partial w}{\partial z}=0
\end{gathered}
$$

where $u$ and $v$ are the eastward and northward horizontal velocities, respectively; $w$ is vertical velocity and $p$ is pressure; here $\rho_{0}$ denotes constant density and $f=f_{0}+\beta y$ is the local Coriolis parameter due to earth rotation.

Considering energy conservation in fluid and introducing the free surface height $h$, then the boundary conditions for the vertical velocity $w$ are given by

$$
\frac{\partial p}{\partial t}+u \frac{\partial p}{\partial x}+v \frac{\partial p}{\partial y}+\left.\rho_{0} g w\right|_{z=0}=0 ;\left.w\right|_{z=h}=\frac{d h}{d t}
$$


In fluid dynamics, the quasi-geostrophic motion refers to nearly geostrophic flows where the advective derivative terms in the momentum equation are an order of magnitude smaller than the Coriolis and the pressure gradient forces, which suggest that the pressure field acts as a stream function for the horizontal motion. Thus, by introducing quasigeostrophic approximation, differentiating the Equation (2) with respect to $y$ and the Equation (3) with respect to $x$, then subtracting the latter from the former yields the quasigeostrophic vorticity equation. Notably, the geostrophic wind is independent of height in homogeneous fluid, then integrating vertically from the bottom to free surface, and using quasi-geostrophic scaling so that geostrophic relative vorticity is far less than planet vorticity in fluid to obtain

$$
\frac{\partial \xi_{g}}{\partial t}+u_{g} \frac{\partial \xi_{g}}{\partial x}+v_{g} \frac{\partial \xi_{g}}{\partial y}+\beta v_{g}=\frac{f_{0}}{H_{0}} \frac{d h}{d t}+\frac{f_{0}}{\rho_{0} g H_{0}}\left(\frac{\partial p}{\partial t}+u_{g} \frac{\partial p}{\partial x}+v_{g} \frac{\partial p}{\partial y}\right)
$$

where $\xi_{g}$ defines the geostrophic relative vorticity, $u_{g}$ and $v_{g}$ are the geostrophic wind in the horizontal $x$ and $y$ direction, respectively. $H_{0}$ is the depth of fluid with a positive value from the free surface to the bottom.

Introducing the geostrophic stream function $\psi$, and considering a pressure forcing that moves at a basic wind shear speed $\bar{u}=\bar{u}(y)$ in the $x$-direction, which gives

$$
\psi=\frac{g h}{f_{0}}, u_{g}=\bar{u}-\frac{\partial \psi}{\partial y}, v_{g}=\frac{\partial \psi}{\partial x}, \xi_{g}=-\frac{\partial \bar{u}}{\partial y}+\nabla^{2} \psi, \nabla^{2}=\frac{\partial^{2}}{\partial x^{2}}+\frac{\partial^{2}}{\partial y^{2}}, L_{0}^{2}=\frac{f_{0}^{2}}{g H_{0}}
$$

Substituting Equation (7) into (6) and considering the balance between nonlinear, dispersive, and pressure forcing, a single non-dimensional quasi-geostrophic stream function equation in one unknown $\psi$ is given by

$$
\begin{gathered}
\left(\frac{\partial}{\partial t}+\bar{u} \frac{\partial}{\partial x}\right) \nabla^{2} \psi-\epsilon \frac{\partial \psi}{\partial t}+J\left(\psi, \nabla^{2} \psi\right)+\left(\beta-\epsilon \bar{u}-\frac{d^{2} \bar{u}}{d y^{2}}\right) \frac{\partial \psi}{\partial x} \\
=\epsilon\left(\frac{\partial p}{\partial t}+\left(\bar{u}-\frac{\partial \psi}{\partial y}\right) \frac{\partial p}{\partial x}+\frac{\partial \psi}{\partial x} \frac{\partial p}{\partial y}\right)
\end{gathered}
$$

where Jacobian operator $J(a, b)=a_{x} b_{y}-a_{y} b_{x}$, the non-dimensional small parameter $\epsilon=\frac{L^{2}}{L_{0}^{2}}$, the ratio of non-geostrophic horizontal perturbation scale to Rossby deformation radius, denotes vorticity dominate pressure field. Along with meridional boundary conditions $\left.\psi\right|_{y=y_{1}}=0$ and $\left.\psi\right|_{y=y_{2}}=0$.

Introducing the multiple space and time scales with the coordinate transformation $\xi=\epsilon^{\frac{1}{2}}(x-c t), T=\epsilon^{\frac{3}{2}} t$ and $y=y$, where $c$ represents phase speed of the perturbation, as usual for the derivation of $\mathrm{KdV}$ type equations. Taking the substitution of the multi-scale transformation into Equation (8) yields

$$
\begin{gathered}
\frac{\partial}{\partial \xi}\left[(\bar{u}-c) \frac{\partial^{2} \psi}{\partial y^{2}}+\left(\beta-\epsilon(\bar{u}-c)-\frac{d^{2} \bar{u}}{d y^{2}}\right) \psi\right]+\epsilon^{2} \frac{\partial^{3} \psi}{\partial T \partial \xi^{2}} \\
+\epsilon\left[\frac{\partial}{\partial T}\left(\frac{\partial^{2} \psi}{\partial y^{2}}-\epsilon \psi\right)+(\bar{u}-c) \frac{\partial^{3} \psi}{\partial \xi^{3}}\right]+\left(\frac{\partial \psi}{\partial \xi} \frac{\partial^{3} \psi}{\partial y^{3}}-\frac{\partial \psi}{\partial y} \frac{\partial^{3} \psi}{\partial \xi}\right) \\
+\epsilon\left(\frac{\partial \psi}{\partial \xi} \frac{\partial^{3} \psi}{\partial \xi^{2} \partial y}-\frac{\partial \psi}{\partial y} \frac{\partial^{3} \psi}{\partial \xi \partial y^{2}}\right)=\epsilon^{2} \frac{\partial p}{\partial t}+\epsilon\left(\bar{u}-c-\frac{\partial \psi}{\partial y}\right) \frac{\partial p}{\partial \xi}+\epsilon \frac{\partial \psi}{\partial \xi} \frac{\partial p}{\partial y}
\end{gathered}
$$

Expanding $\psi$ and $p$ into a suitable power series in parameter $\epsilon$, namely,

$$
\psi=\sum_{k=1}^{\infty} \epsilon^{k} \psi_{k}(\xi, y, T), p=\sum_{k=1}^{\infty} \epsilon^{k} P_{k}(\xi, y, T)
$$


Substituting Equation (10) into (9), and it requires all the coefficients of different powers of $\epsilon$ to be zero. Then vanishing the coefficients of $O(\epsilon)$ and $O\left(\epsilon^{2}\right)$ gives the following first-order linear partial differential equation

$$
\frac{\partial}{\partial \xi}\left[(\bar{u}-c) \frac{\partial^{2} \psi_{1}}{\partial y^{2}}+\left(\beta-\frac{d^{2} \bar{u}}{d y^{2}}\right) \psi_{1}\right]=0
$$

and the second-order nonlinear partial differential equation

$$
\begin{gathered}
\frac{\partial}{\partial \xi}\left[(\bar{u}-c) \frac{\partial^{2} \psi_{2}}{\partial y^{2}}+\left(\beta-\frac{d^{2} \bar{u}}{d y^{2}}\right) \psi_{2}\right]+\frac{\partial^{3} \psi_{1}}{\partial y^{2} \partial T}+(\bar{u}-c) \frac{\partial^{3} \psi_{1}}{\partial \xi^{3}} \\
+\frac{\partial \psi_{1}}{\partial \xi} \frac{\partial^{3} \psi_{1}}{\partial y^{3}}-\frac{\partial \psi_{1}}{\partial y} \frac{\partial^{3} \psi_{1}}{\partial \xi \partial y^{2}}=(\bar{u}-c) \frac{\partial P_{1}}{\partial \xi}
\end{gathered}
$$

respectively.

Obviously, Equation (11) is linear so it can be solved by the usual variable separation approach. Suppose $\psi_{1}$ has a variable separation solution of the form $\psi_{1}=A(\xi, T) G(y)$. The substitution of this expression into (11) produces

$$
\frac{d^{2} G}{d y^{2}}+\frac{\beta-\frac{d^{2} \bar{u}}{d y^{2}}}{\bar{u}-c} G=0
$$

For the continuous velocity profile, it is not difficult to derive a necessary condition for the occurrence of instability. The flow profile have an inflexion point with a maximum speed and inflexion-point instability can occur in fluid, which can contribute to the formation and maintenance of vortex. Using the meridional boundary condition, the solution of (13) may be obtained as

$$
G(y)=\sin \left(\frac{y-y_{1}}{y_{2}-y_{1}} n \pi\right) ; c=\bar{u}-\frac{\beta}{n \pi}\left(y_{2}-y_{1}\right) ; n=1,2, \ldots
$$

Note that we have chosen the free wave to have the same structure as the forced wave, which corresponds to the wind stress forcing, in order to maximize the effect of pressure forcing on the free waves. It is assumed to be of the form $P_{1}=F(\xi, T) G(y)$. To find the amplitude of the perturbation, $A(\xi, \tau)$, we have to proceed to the next order problem. Substituting this transformation into (12), we have

$$
\begin{gathered}
\frac{\partial}{\partial \xi}\left[(\bar{u}-c) \frac{\partial^{2} \psi_{2}}{\partial y^{2}}+\left(\beta-\frac{d^{2} \bar{u}}{d y^{2}}\right) \psi_{2}\right]+\frac{d^{2} G}{d y^{2}} \frac{\partial A}{\partial T}+(\bar{u}-c) G \frac{\partial^{3} A}{\partial \xi^{3}} \\
+\left(G \frac{d^{3} G}{d y^{3}}-\frac{d G}{d y} \frac{d^{2} G}{d y^{2}}\right) A \frac{\partial A}{\partial \xi}=(\bar{u}-c) G \frac{\partial F}{\partial \xi}
\end{gathered}
$$

Multiplying (15) by $G$, and integrating the result with respect to $y$ from $y_{1}$ to $y_{2}$, considering the meridional boundary condition, then a standard KdV equation with a forcing term is derived immediately

$$
\frac{\partial A}{\partial T}+\alpha A \frac{\partial A}{\partial \xi}+\gamma \frac{\partial^{3} A}{\partial \xi^{3}}=\gamma \frac{\partial F}{\partial \xi}
$$

where the coefficients satisfy

$$
\begin{gathered}
\alpha=\frac{I_{1}}{I_{0}}, \gamma=\frac{I_{2}}{I_{0}} \\
I_{0}=\int_{y_{1}}^{y_{2}} \frac{d^{2} G}{d y^{2}} \frac{G}{\bar{u}-c} d y
\end{gathered}
$$




$$
\begin{gathered}
I_{1}=\int_{y_{1}}^{y_{2}} \frac{G}{\bar{u}-c}\left(G \frac{d^{3} G}{d y^{3}}-\frac{d G}{d y} \frac{d^{2} G}{d y^{2}}\right) d y \\
I_{2}=\int_{y_{2}}^{y_{1}} G^{2} d y
\end{gathered}
$$

Here, $G=G(y)$ satisfy (14).

\section{Solutions of the Forced KdV Equation}

The forced KdV Equation (16) can be transformed into the following form

$$
\frac{\partial B}{\partial T}+B \frac{\partial B}{\partial \xi}+\gamma \frac{\partial^{3} B}{\partial \xi^{3}}=\alpha \gamma \frac{\partial F}{\partial \xi}
$$

by letting $A=\alpha B$. Introducing the dependent variable transformation

$$
B=12 \gamma \frac{\partial^{2} \ln \tau}{\partial \xi^{2}}, F=\frac{f}{\tau}
$$

By introducing the bilinear method ([22]), the forced KdV Equation (18) can be transformed into a bilinear equation in $\tau$ function form as follows

$$
D_{\xi}\left(D_{T}+\gamma D_{\xi}^{3}\right)(\tau \cdot \tau)=\frac{\alpha}{6} f \tau
$$

where the bilinear operator $D_{\tilde{\zeta}}^{m} D_{\chi}^{n}$ are defined by

$$
D_{\xi}^{m} D_{\chi}^{n} a \cdot b=\left.\left(\frac{\partial}{\partial \xi}-\frac{\partial}{\partial \xi^{\prime}}\right)^{m}\left(\frac{\partial}{\partial \chi}-\frac{\partial}{\partial \chi^{\prime}}\right)^{n} a(\xi, \chi) b\left(\xi^{\prime}, \chi^{\prime}\right)\right|_{\xi^{\prime}=\xi, \chi^{\prime}=\chi}
$$

By selecting the forcing functions $F$, we seek two particular solutions (derived using Hirota's method as in [10-12]) to the nonlinear model Equation (16) and stream-function Equation (8) as follows:

(1) When choosing the vortex type pressure forcing term $F$ given as follows

$$
\left.F=\frac{12 k}{\alpha}\left(k^{3}(4 \gamma-\delta)-\frac{\partial a(T)}{\partial T}\right) \operatorname{sech}^{2}\left(k \xi-\delta k^{3} T\right)-a(T)\right)
$$

where there is an arbitrary function $a(T)$, thus, different types of waves can be excited by different external forcing sources.

By selecting $a(T)=b_{2} \arctan (\mu T)+b_{1} T+b_{0}$, with $b_{2}, b_{1}, b_{0}, \delta$ and $\mu$ being constants, we can obtain a special type wave solution

$$
A=12 \alpha \gamma k^{2} \operatorname{sech}^{2}\left(k \xi-\delta k^{3} T-b_{2} \arctan (\mu T)-b_{1} T-b_{0}\right)
$$

Its behavior and velocity field of $A$ defined above are shown in Figure 1 (Left).

Considering Equations (14) and (22), one possible approximate stream-function solution to the Equation (8) is obtained in the following

$$
\psi=-\bar{u} y+12 \epsilon \alpha \gamma k^{2} \sin \left(\frac{y-y_{1}}{y_{2}-y_{1}} n \pi\right) \operatorname{sech}^{2}\left(\epsilon^{\frac{1}{2}} k(x-c t)-\epsilon^{\frac{3}{2}} \delta k^{3} t-a(T)\right)
$$

This solution (23) shows that various multi-pole vortex structure can be excited by pressure forcing source in shear fluid (Figure 2). 

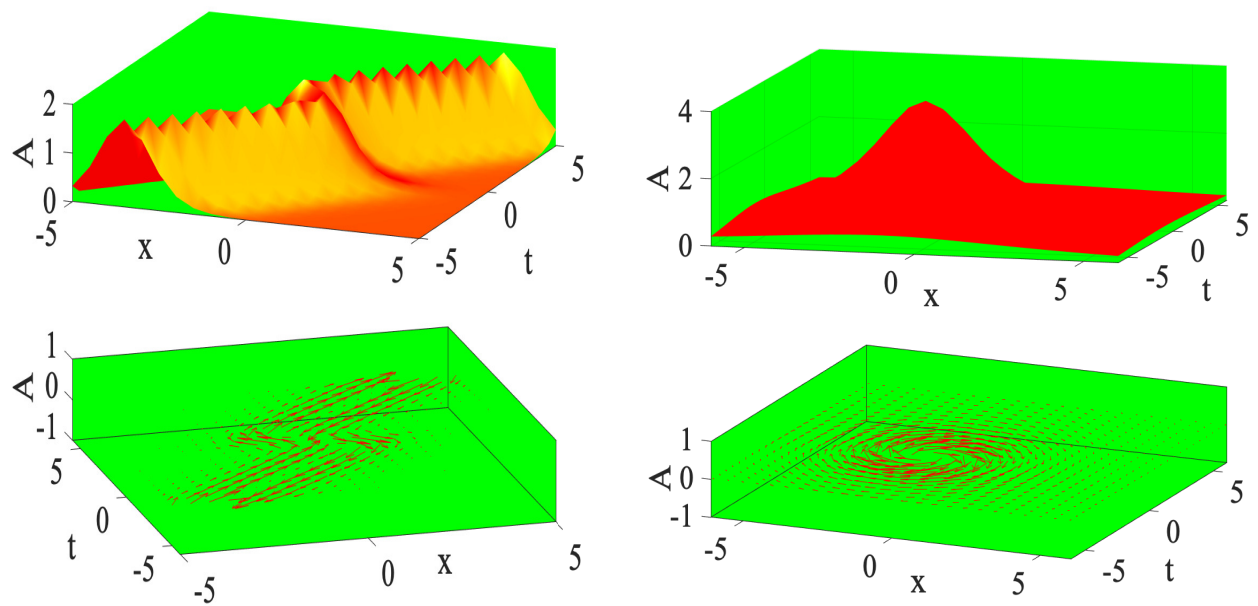

Figure 1. The wave expressed by solution (22) (Left) and a lump-type vortex expressed by solution (25) (Right) with parameters chosen as: $k=1, \alpha=0.2, \gamma=0.25, \delta=4, \mu=3, a_{2}=1, a_{1}=1$, $a_{0}=5, b_{2}=-1, b_{1}=-3, b_{0}=0$ and $d=4$.

(2) When choosing the pressure forcing source $F$ reads

$$
\begin{gathered}
F=\frac{12 a_{0}}{\alpha}\left(\frac{6 \gamma\left(a_{0}+1\right)}{p_{1}^{2}}-\frac{8 \gamma\left(\xi+a_{2}\right)^{2}}{p_{2}^{2} p_{1}^{3}}-\frac{\left(T+a_{1}\right) p_{2}^{2}}{\left(\xi+a_{2}\right) p_{1}}\right. \\
\left.-\frac{\left(T+a_{1}\right) p_{1}^{3}}{\left(\xi+a_{2}\right)^{3}} \arctan \left(p_{1}\right)+\frac{\partial m(T)}{a_{0} \partial T}\right)
\end{gathered}
$$

where $a_{2}, a_{1}, a_{0}$ and $d$ are arbitrary constants and $m(T)$ is arbitrary functions of $t ; p_{1}$ and $p_{2}$ defined by $p_{1}=\left(\xi+a_{2}\right)^{2}+\left(T+a_{1}\right)^{2}+d$ and $p_{2}=\frac{\xi+a_{2}}{\left(T+a_{1}\right)^{2}+d}$.

By using perturbation method, another type analytic solution to the forced $\mathrm{KdV}$ equation are obtained as follows

$$
A=12 \alpha \frac{a_{0} \gamma}{\left(\xi+a_{2}\right)^{2}+\left(T+a_{1}\right)^{2}+d}
$$

Obviously, a lump type vortex solution from solution (25) can be obtained by choosing suitable parameters. Noticing that the arbitrary function $m(T)$ in (24) is independent with the solution $A$; therefore, by selecting $m(T)=b_{2} \sin \left(b_{1} t+b_{0}\right)$ or $m(T)=b_{2} \arctan \left(\left(T+a_{1}\right)^{2}+b_{0}\right)$, the different pressure forcing source can excite the same type vortex solution (Figure 1 Right).

By considering Equations (14) and (25), one possible approximate stream-function solution to (8) reads

$$
\psi=-\bar{u} y+\frac{12 \alpha a_{0} \gamma}{\left(\epsilon^{1 / 2}(x-c t)+a_{2}\right)^{2}+\left(\epsilon^{3 / 2} t+a_{1}\right)^{2}+d} \sin \left(\frac{y-y_{1}}{y_{2}-y_{1}} n \pi\right)
$$

The about exact solution (26) shows that solitary wave and vortex multi-pole structure can be excited by various pressure forcing source in shear fluid (Figure 3). 
(a)
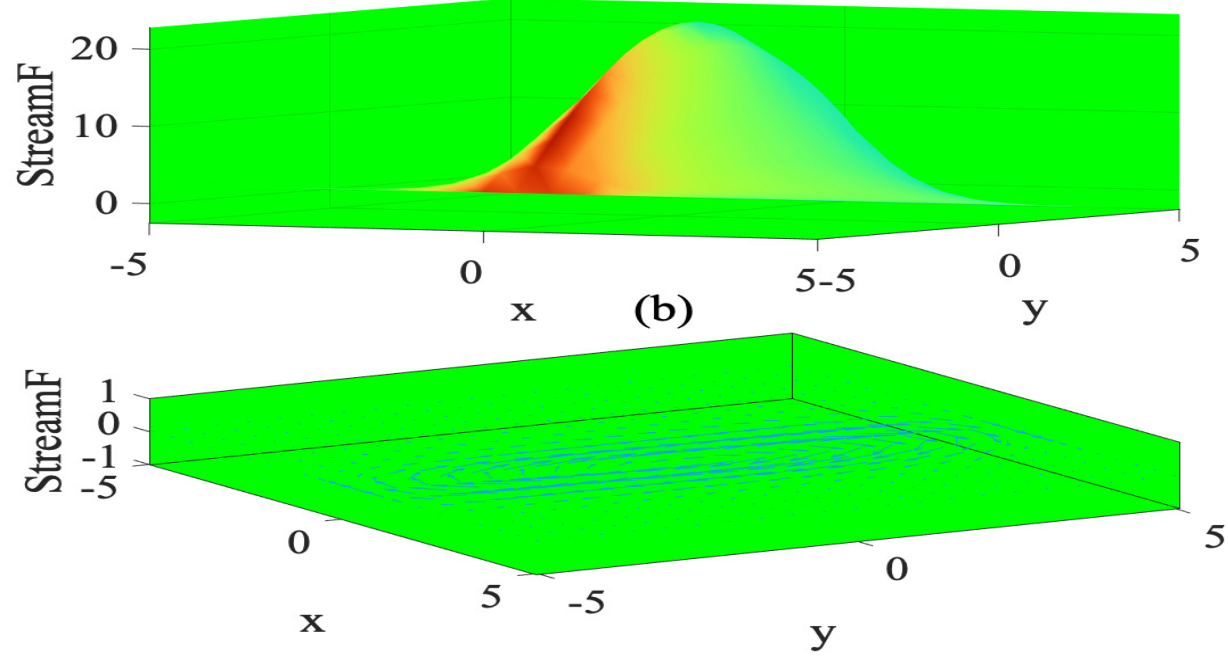

(c)
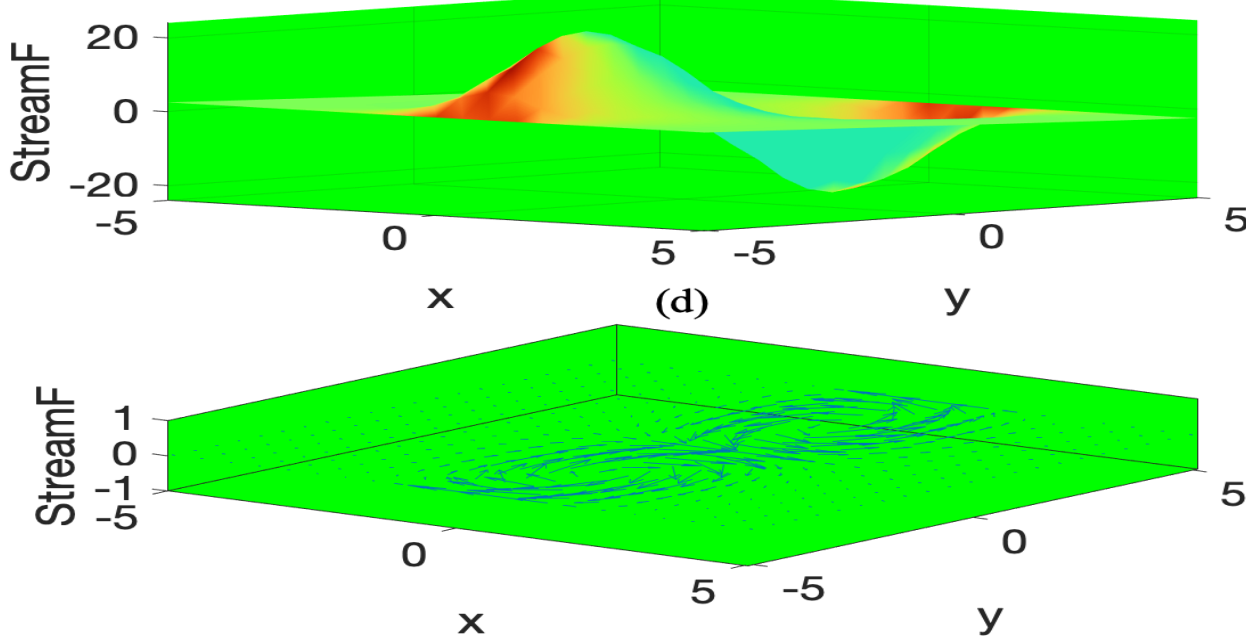

(e)

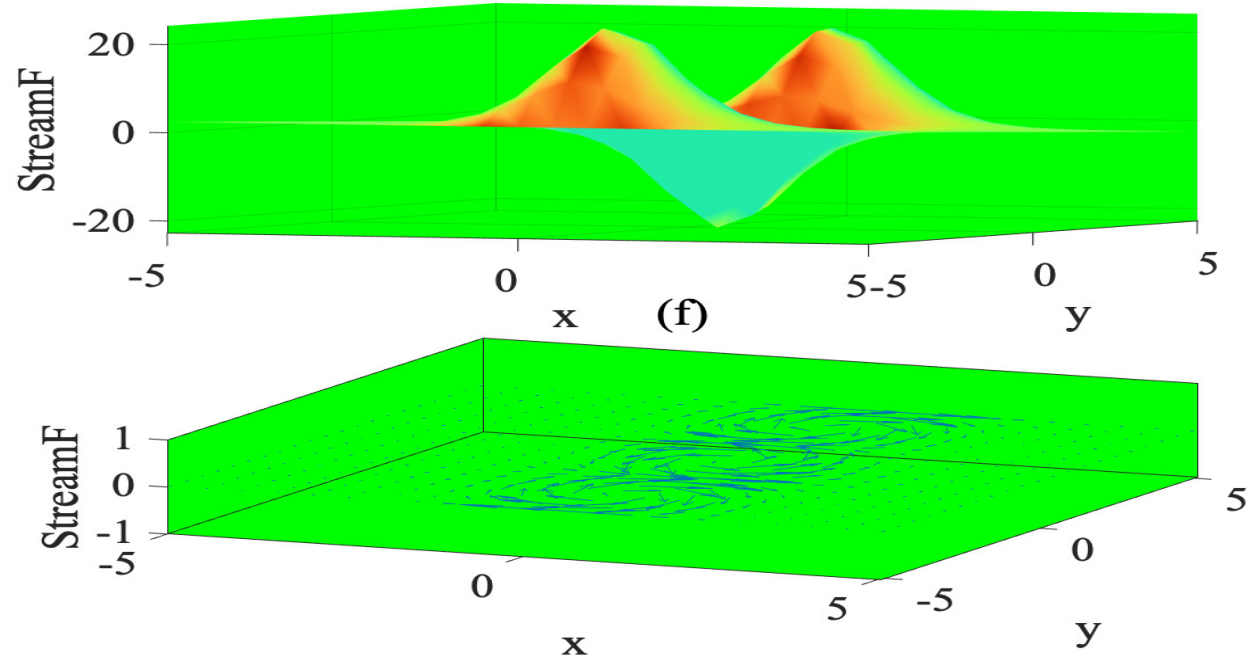

Figure 2. The Soliton-type vortex monopole $(\mathbf{a}, \mathbf{b}$, with parameter $\mathrm{n}=1)$, vortex dipole $(\mathbf{c}, \mathbf{d}$, with parameter $n=2)$, and vortex tripole $(\mathbf{e}, \mathbf{f}$, with parameter $n=3)$, expressed by stream function solution (23) with its other parameters chosen as: $\epsilon=0.25, c=0.5, \bar{u}=0.5, k=1, \alpha=2, \gamma=2.5$, $\delta=4, \mu=3, T=0.25, b_{2}=-1, b_{1}=-3, b_{0}=1, y_{1}=-5$ and $y_{2}=5$. 
(a)

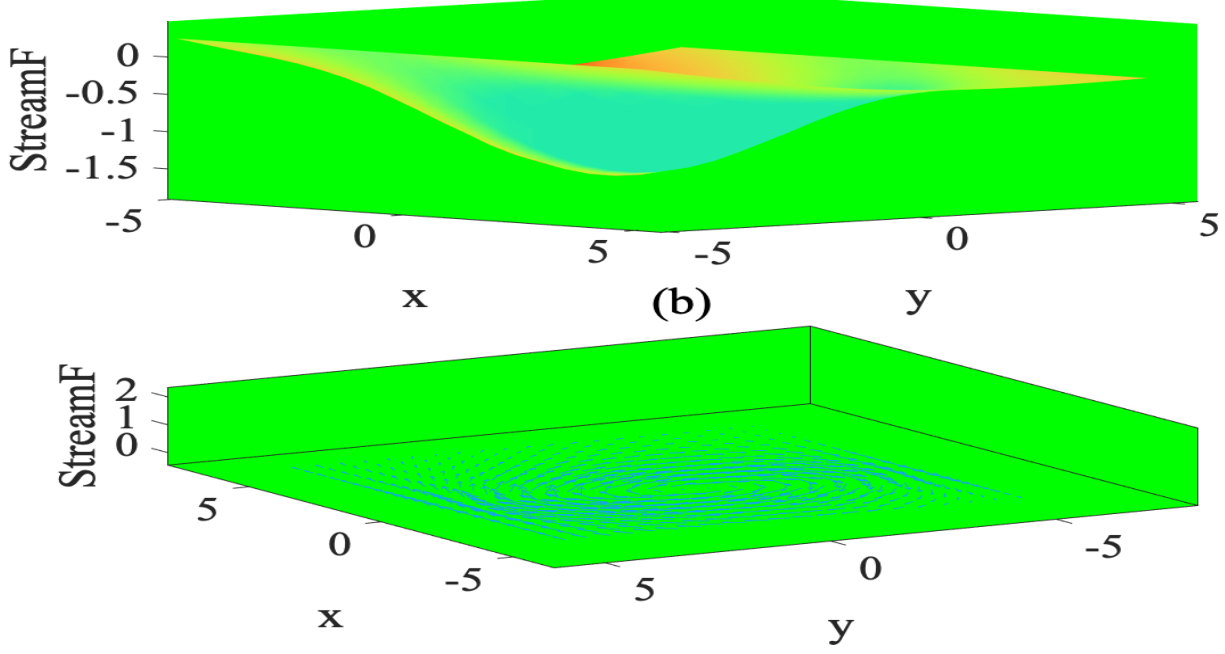

(c)

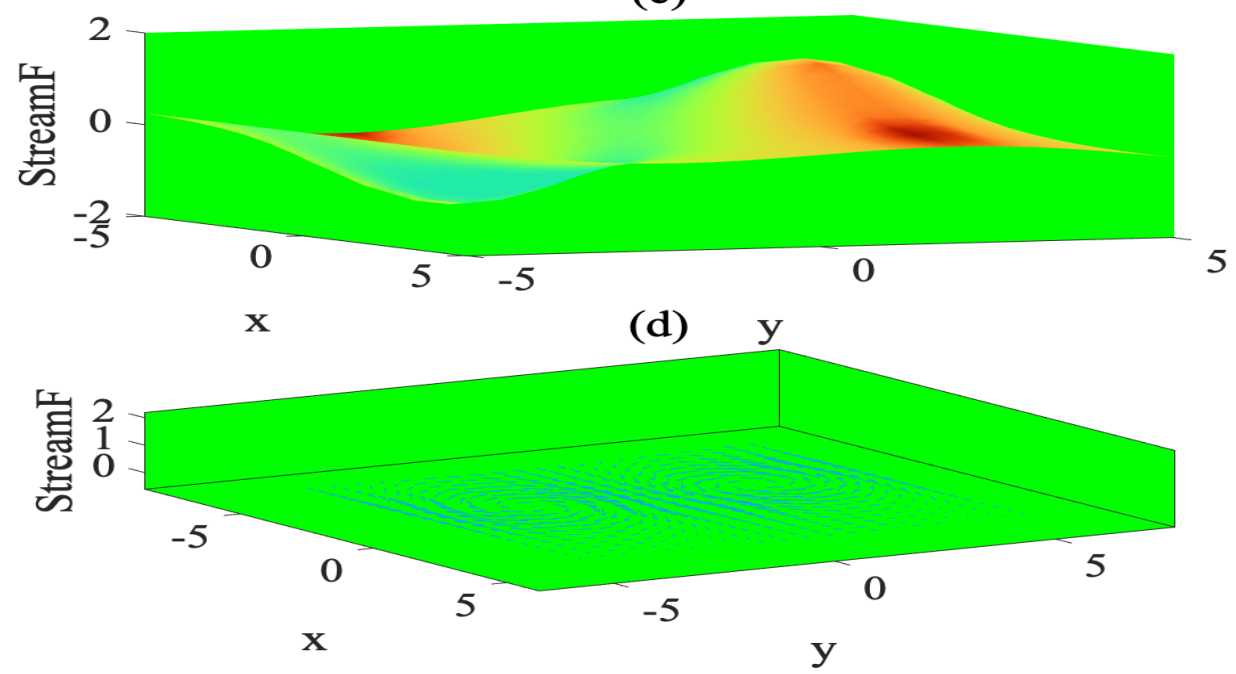

(e)

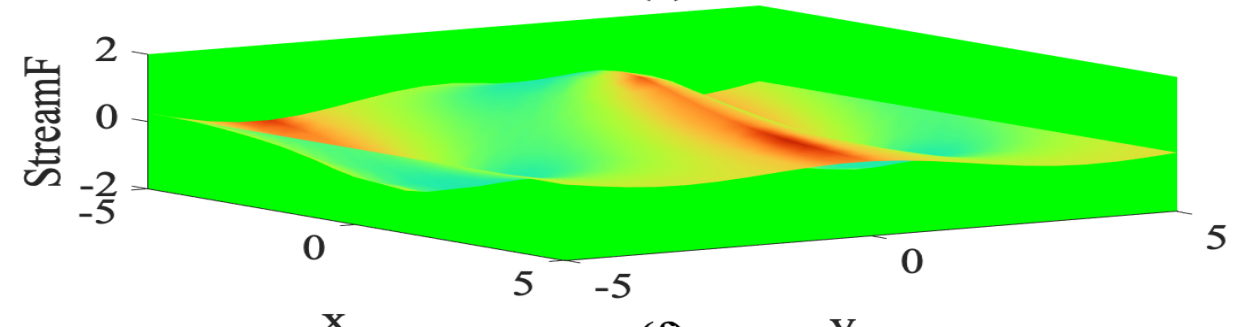

$\mathbf{X}$
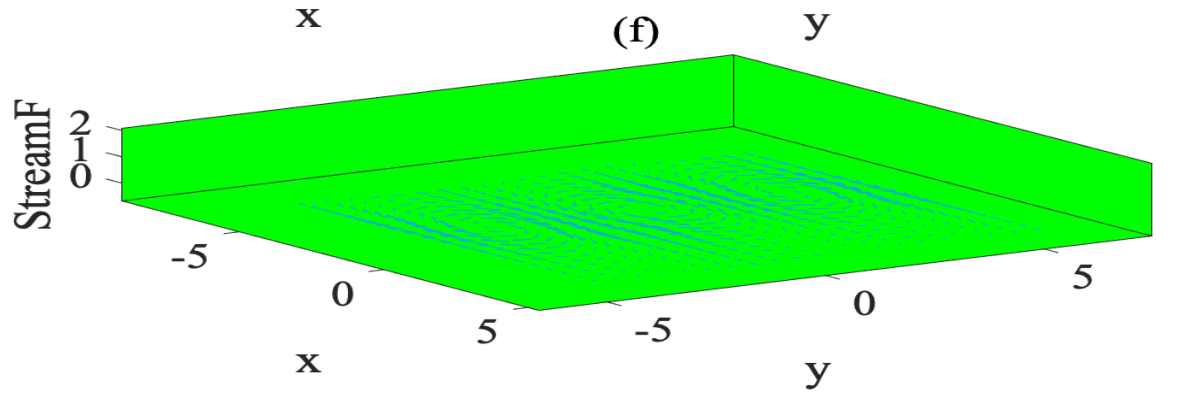

Figure 3. The lump-type vortex monopole $(\mathbf{a}, \mathbf{b}$, with parameter $n=1)$, vortex dipole $(\mathbf{c}, \mathbf{d}$, with parameter $n=2)$, and vortex tripole (e,f, with parameter $n=3)$, expressed by vortex solution (26) with its other parameters chosen as: $\epsilon=0.25, c=0.5, \bar{u}=0.5, \alpha=2, \gamma=2.5, \delta=5, T=0.25, a_{2}=1$, $a_{1}=1, a_{0}=5, d=4, y_{1}=-5$ and $y_{2}=5$. 


\section{Discussion and Conclusions}

In this present work, we stress that a mathematical model, building from the perspective of energy conversation for the first time, has been applied to derive the forced $\mathrm{KdV}$ equation. By the Hirota's bilinear method, the presented solutions from the fKdV equation reflect the significant nonlinear relationship between the pressure disturbance and cyclone genesis. It may be mentioned that vortex solutions can be obtained through Hirota's bilinear methods by assigning particular parameters, which represents various pressure waves and pressure disturbance forcing source. We hope that this work may initiate a powerful thinking to investigate tropical cyclone formation forced by tropical pressure disturbance and pressure waves. Notably, here the proposed method can be applied to derive the higher dimensional and higher-order nonlinear evolution equations from other mathematical model. Thus, a huge variety of higher dimensional nonlinear evolution equations with or without a forcing term help to understand the field of nonlinear wave and vortex excitations in atmosphere.

Author Contributions: Conceptualization, Z.-L.L. and J.-Q.L.; writing—original draft preparation, J.-Q.L. and Z.-L.L.; writing-review and editing, Z.-L.L. All authors have read and agreed to the published version of the manuscript.

Funding: This research was funded by the National Key Basic Research and Development Plan of China (No. 2015CB953900), National Natural Science Foundation of China (No. 40775069), Open Project Fund of Guangdong Provincial Key Laboratory of Regional Numerical Weather Prediction, CMA (No. J202009), Heavy Rain and Drought-Flood Disasters in Plateau and Basin Key Laboratory of Sichuan Province (No. SZKT202005). The APC was funded by Z.-L.L.

Institutional Review Board Statement: Not applicable.

Informed Consent Statement: Not applicable.

Data Availability Statement: Not applicable.

Acknowledgments: The authors are very grateful to the editor and anonymous reviewers for their help.

Conflicts of Interest: The authors declare no conflict of interest.

\section{References}

1. Hirota, R. Exact n-soliton solutions of the wave equation of long waves in shallow water and in nonlinear lattices. J. Math. Phys. 1973, 14, 810-814. [CrossRef]

2. Hirota, R. The Direct Method in Soliton Theory; Cambridge University Press: Cambridge, UK, 2004.

3. Ablowitz, M.J.; Clarkson, P.A. Solitons, Nonlinear Evolution Equations and Inverse Scattering Transform; Cambridge University Press: Cambridge, UK, 1991.

4. Ma, W. Exact one-periodic and two-periodic wave solutions to Hirota bilinear equations in $(2+1)$ dimensions. Mod. Phys. Lett. A 2009, 24, 1677-1888. [CrossRef]

5. Ma, W. Bilinear equations and resonant solutions characterized by bell polynomials. Rep. Math. Phys. 2013, 72, 41-56. [CrossRef]

6. Ma, W.X. N-soliton solutions and the hirota conditions in (2+1)-dimensions. Opt. Quantum Electron. 2020, 52, 511-512. [CrossRef]

7. Chen, F.; Zhang, H.Q. Rogue waves on the periodic background in the higher-order modified Korteweg-de Vries equation. Mod. Phys. Lett. B 2021, 35, 2150081. [CrossRef]

8. Gao, X.; Zhang, H.Q. Rogue waves for the Hirota equation on the Jacobi elliptic cn-function background. Nonlinear Dyn. 2020, 101, 1159-1168. [CrossRef]

9. Chen, J.B.; Pelinovsky, D.E. Rogue periodic waves of the modified KdV equation. Nonlinearity 2018, 31, 1955-1980. [CrossRef]

10. Zhao, J.; Guo, B. Analytic solutions to forced KdV equation. Commun. Theor. Phys. 2009, 52, $279-283$.

11. Salas, A.H. Computing solutions to a forced kdv equation. Nonlinear Anal. Real World Appl. 2011, 12, 1314-1320. [CrossRef]

12. Gandarias, M.L.; Bruzon, M.S. Some conservation laws for a forced KdV equation. Nonlinear Anal. Real World Appl. 2012, 13, 2692-2700. [CrossRef]

13. Ali, R.; Saha, A.; Chatterjee, P. Analytical electron acoustic solitary wave solution for the forced KdV equation in super thermal plasmas. Phys. Plasmas 2017, 24, 122106. [CrossRef]

14. Amiya, D.; Uttam, K.M. Integrability, bilinearization, solitons and exact three wave solutions for a forced Korteweg-de Vries equation. Commun. Nonlinear Sci. Numer. Simul. 2021, 102, 105936. 
15. Mandi, L.; Mondal, K.K.; Chatterjee, P. Analytical solitary wave solution of the dust ion acoustic waves for the damped forced modified Korteweg-de Vries equation in q-nonextensive plasmas. Eur. Phys. J. Spec. Top. 2019, 228, 2753-2768. [CrossRef]

16. Muller, C.J.; Romps, D.M. Acceleration of tropical cyclogenesis by self-aggregation feedbacks. Proc. Natl. Acad. Sci. USA 2018, 115, 2930-2935. [CrossRef] [PubMed]

17. Emanuel, K. 100 years of progress in tropical cyclone research. A Century of Progress in Atmospheric and Related Sciences: Celebrating the American Meteorological Society Centennial. Meteorol. Monogr. Am. Meteorol. Soc. 2018, 59, 15.1-15.68.

18. Davis, C.A. The formation of moist vortices and tropical cyclones in idealized simulations. J. Atmos. Sci. 2015, 72, 3499-3516. [CrossRef]

19. Frank, W.M.; Roundy, P.E. The role of tropical waves in tropical cyclogenesis. Mon. Weather Rev. 2006, 134, 2397-2417. [CrossRef]

20. Ritchie, E.; Holland, G. Large-scale patterns associated with tropical cyclogenesis in the western Pacific. Mon. Weather Rev. 1999, 127, 2027-2043. [CrossRef]

21. Narenpitak, P.; Bretherton, C.S.; Khairoutdinov, M.F. The role of multiscale interaction in tropical cyclogenesis and its predictability in near-global aquaplanet cloud-resolving simulations. J. Atmos. Sci. 2020, 55, 3177-3196. [CrossRef]

22. Yuan, P.; Qi, J.; Li, Z.; An, H. General M-lumps, T-breathers, and hybrid solutions to (2 + 1)-dimensional generalized KDKK equation. Chin. Phys. B 2021, 30, 040503. [CrossRef] 